\title{
Polar Surface Induced Growth of Semiconducting and Piezoelectric Oxide Novel Nanostructures
}

\author{
Zhong Lin (ZL) Wang \\ School of Material Science and Engineering, Georgia Institute of Technology \\ Atlanta GA 30332-0245, USA \\ * E-mail: zhong.wang@mse.gatech.edu
}

Quasi-one-dimensional (1D) nanostructures (nanowires, nanobelts and nanorods) are the forefront nanomateirals for nanotechnology. Oxide nanostructures have been synthesized for a wide range of semiconducting oxides [1] that are potential building blocks for constructing numerous nanodevices. Using the technique demonstrated for measuring the mechanical properties of nanotubes [2,3], the mechanical and field emission properties of the oxide nanobelts have been characterized. Field effect transistors [4], ultra-sensitive nano-size gas sensors [5], nanoresonators and nanocantilevers [6] have been fabricated using nanobelts.

Among all of the oxide nanostructures we have investigated, $\mathrm{ZnO}$ is very unusual. The two important characteristics of the wurtzite structured $\mathrm{ZnO}$ are the non-central symmetry and the polar surfaces. The structure of $\mathrm{ZnO}$ can be described as a number of alternating planes composed of tetrahedrally coordinated $\mathrm{O}^{2-}$ and $\mathrm{Zn}^{2+}$ ions, stacked alternatively along the $c$-axis. The oppositely charged ions produce positively charged (0001)-Zn and negatively charged (000-1)-O polar surfaces, resulting in a normal dipole moment and spontaneous polarization along the $c$-axis. Growth of (0001) facets dominated, freestanding, piezoelectric zinc oxide $(\mathrm{ZnO})$ nanostructures is challenged by the divergence of the surface energy due to intrinsic polarization. By controlling growth kinetics, we show the success of growing nanobelt-based novel structures whose surfaces are dominated by the polarized $\pm(0001)$ facets. Owing to the positive and negative ionic charges on the zinc- and oxygen-terminated $\pm(0001)$ surfaces, respectively, a spontaneous polarization is induced across the nanobelt thickness. As a result, helical nanostructures and nanorings are formed by rolling up single-crystal nanobelts. This phenomenon is attributed to a consequence of minimizing the total energy contributed by spontaneous polarization and elasticity. The polar surfaces give raise a few interesting growth features, such as the formations of nanosprings [7], nanorings [8], nanobows [9] and nanohelices [10]. These nanostructure are semiconductive and piezoelectric and have potential applications as nano-scale sensors, traducers, and actuators. The polar surface dominated $\mathrm{ZnO}$ nanobelts are likely to be an ideal system for understanding piezoelectricity and polarization induced ferroelectricity at nano-scale; and they could have applications as one-dimensional nano-scale sensors, transducers and resonators.

[1] Z.W. Pan, Z.R. Dai and Z.L. Wang, Science, 209 (2001) 1947.

[2] P. Poncharal, Z.L. Wang, D. Ugarte and W.A. de Heer, Science, 283 (1999) 1513.

[3] R.P. Gao, Z.L. Wang, Z.G. Bai, W. de Heer, L. Dai and M. Gao, Phys. Rev. Letts., 85 (2000) 622.

[4] M. Arnold, P. Avouris, Z.L. Wang,. Phys. Chem. B, 107 (2002) 659.

[5] E. Comini, G. Faglia, G. Sberveglieri, Zhengwei Pan, Z. L. Wang Applied Physics Letters, 81 (2002) 1869.

[6] W. Hughes and Z.L. Wang, Appl. Phys. Letts., 82 (2003) 2886.

[7] X.Y. Kong and Z.L. Wang, Nano Letters, 2 (2003) 1625 + cover. 
[8] X.Y. Kong, Y. Ding, R.S. Yang, Z.L. Wang, Science, 303 (2004) 1348.

[9] W.L. Hughes and Z.L. Wang, J. Am. Chem. Soc., 126 (2004) 670

[10] P.X. Gao, Y. Ding, W.J. Mai, W.L. Hughes, C.S. Lao and Z.L. Wang, Science, 309 (2005) 1700.

[11] Thanks the support from NSF, DARPA, NASA and Airforce.

[12] for details see: http://www.nanoscience.gatech.edu/zlwang/

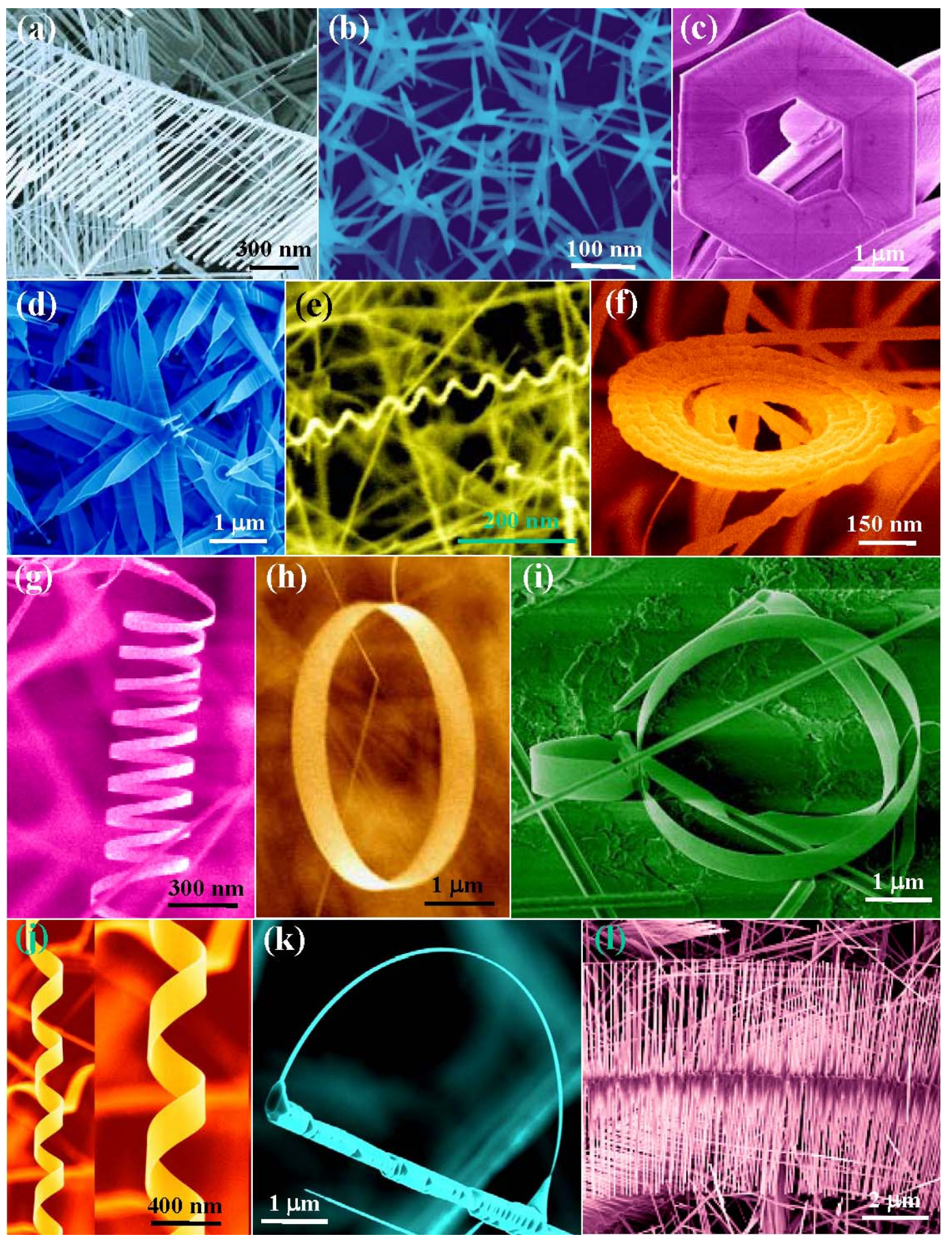

FIG.1 Nanostructures of Zinc Oxide formed due the presence of polar surfaces. These unique nanostructures were grown by a vapor-solid process 